\title{
Early changes in right ventricular longitudinal function in chronic asymptomatic alcoholics revealed by two-dimensional speckle tracking echocardiography
}

Sisi Meng ${ }^{1}$, Lijuan Guo ${ }^{2}$ and Guangsen $\mathrm{Li}^{1 *}$

\begin{abstract}
Background: Heart ventricular dysfunction has been characterized as reduced longitudinal function of the right ventricle (RV), and is associated with chronic alcohol abuse. This study investigated the use of two-dimensional speckle tracking echocardiography (2DSTE) to assess the longitudinal systolic and diastolic RV function of patients with alcoholic myocardial damage.
\end{abstract}

Methods: We stratified 92 asymptomatic alcoholic men into three groups of increasing alcohol intake, Groups A-C. Thirty age-matched normal adult men served as the control group. Conventional echocardiography and tricuspid annulus peak systolic excursion (TAPSE) parameters were obtained. 2DSTE parameters were recorded from an apical 4-chamber view of the RV free wall. LV peak global longitudinal systolic strain was calculated from segmental averaging of the three apical long-axis views.

Results: In Group C, the RV end diastolic diameter (RVEDD) was dramatically higher than that of Groups A, B and the control, while TAPSE was significantly lower in Group C compared with the other experimental groups. In Group B, the longitudinal early diastolic strain rate (SRe) and late diastolic strain rate (SRa) of the RV free wall, and LV longitudinal strain were significantly lower than that of Group A or the control. In Group C, all the 2DSTE parameters were significantly lower than that of the other groups. A significant negative linear correlation was noted between global RV systolic parameters systolic strain peak (S), peak systolic strain rate (SRs) and TAPSE $\left(r_{1}=-0.84, r_{2}=-0.72\right.$, respectively, $\left.P<0.05\right)$.

Conclusions: Two-dimensional STE provided an effective and non-invasive method to assess the RV longitudinal function of patients with alcoholic myocardial damage. This methodology may be useful for diagnosing, directing treatment, and judging prognosis of alcoholic cardiac damage.

Keywords: Two-dimensional speckle tracking echocardiography, Alcoholics, TAPSE, Right ventricular function

\footnotetext{
*Correspondence: liguangsen09@163.com

${ }^{1}$ Department of Ultrasound, Second Affiliated Hospital of Dalian Medical

University, Dalian 116027, China

Full list of author information is available at the end of the article
} 


\section{Background}

Alcohol is a risk factor of heart disease, and long-term alcohol abuse may have detrimental effects on ventricular function $[1,2]$, possibly leading to alcoholic cardiomyopathy (ACM). Recent studies have indicated that changes in heart dimensions and function are common in alcoholics [3].

Longitudinal LV dysfunction is one of the earliest biomarkers of ventricular damage $[4,5]$. Two-dimensional speckle tracking echocardiography (2DSTE) reportedly can sensitively assess left ventricular (LV) function and predict early pathogenesis associated with myocardial dysfunction $[6,7]$.

Although the prognostic value of right ventricular (RV) systolic and diastolic function has been documented for cardiac symptoms and exercise capacity [8], prior studies using 2DSTE have focused on LV structure and function $[9,10]$, and studies on RV function are few. RV function is difficult to assess quantitatively using traditional imaging because of the thin RV myocardial wall and complex structure of the RV myocardial wall, thus it is usually described only qualitatively $[11,12]$.

The limitations of other echocardiographic techniques are overcome with 2DSTE, as the focus is on endocardial structures interference from the RV wall is avoided. Prior studies have suggested that RV dysfunction can be determined quantitatively by the change in longitudinal movement of the $R V$, relative to that of the normal RV $[13,14]$. Since the subendocardial layer of the myocardium is the most sensitive to alcohol damage [15], we hypothesized that measurements of RV longitudinal deformation could be a sensitive measure of RV function in an alcoholic population [16].

In this setting, we hypothesized that 2DSTE could be used for early evaluation of changes in RV longitudinal function in patients with alcoholic myocardial damage. While there have been several studies of deformation that have touched upon this topic, none have used 2DSTE to study the RV.

\section{Methods}

\section{Patient population}

Ninety-two male asymptomatic alcoholics, 42-65 years old, were examined between June 2009 and March 2013 and served as the experimental group. These patients were categorized into three groups according to the amount and duration of alcohol consumption: Group A, $\geq 90 \mathrm{mg}$ ethanol or $\geq 2-3$ bottles of beer, 3-5 days/week for 5-8 years; Group B, $\geq 90 \mathrm{mg}$ ethanol or $\geq 2-3$ bottles of beer, 3-5 days/week for 9-20 years; and Group C, $\geq 150 \mathrm{mg}$ ethanol or $\geq 4$ bottles of beer, 6-7 days/week for $>10$ years, which met the standard for diagnosis of alcoholic cardiomyopathy (ACM) [17].
Thirty age-matched (40-65 years) healthy male volunteers served as the control group (Group D). Control subjects did not exhibit any cardiovascular abnormalities.

The exclusion criteria included a history of congenital heart disease, hypertension, diabetes, ischemic or vascular heart diseases, rhythm problems and atrial fibrillation, pulmonary hypertension, systemic and metabolic diseases that could adversely affect cardiac structure and function such as valvular heart disease, or using cardiac medications.

All procedures were carried out in accordance with institutional guidelines. The local medical ethics committee (Second Affiliated Hospital of Dalian Medical University, Dalian, China) approved the study, and all patients provided written informed consent before entering the study.

\section{Instruments and methods}

Recordings were obtained with a GE Vingmed Vivid 7 (GE Vingmed Ultrasound, Horten, Norway) scanner equipped with a $1.7-3.4 \mathrm{MHz}$ transducer (M3S probe). Standard echocardiography and 2DSTE measurements of the RV and LV were analyzed off-line using dedicated software (EchoPAC, GE Vingmed Ultrasound).

\section{Two-dimensional and M-mode echocardiographic acquisition}

The examination focused on the measurement of LV and RV dimensions. The end diastolic diameters, ejection fractions, and tricuspid annulus peak systolic excursion (TAPSE) were captured as follows. RV and LV end diastolic diameters (RVEDD and LVEDD, respectively) were measured in the parasternal long-axis view. From the apical four-chamber view, the LV ejection fraction (LVEF) was obtained using a commercially available software program that applied the biplane Simpson's method in the four-chamber view. The RV ejection fraction (RVEF) was calculated from the RV fractional area change.

TAPSE acquisition was performed by M-mode echocardiography in the four-chamber view: an M-mode cursor was steered through the lateral portion of the tricuspid annulus, which delineates the RV atrioventricular plane. The magnitude of the tricuspid valve ring along the M-mode line was determined by measuring the maximal distance between the leading edge of the highest and lowest echoes during each cardiac cycle.

\section{DSTE acquisition}

Two-dimensional dynamic images were recorded for subsequent analyses. High frame rate acquisition was used ( $\geq 100$ frames/s). At least three cardiac cycles were stored for offline analysis. All 2DSTE data were measured by averaging data over three heartbeats.

A crucial part of the analysis was based on the correct adjudication of event timing. For example, end-diastole was defined at the peak $\mathrm{R}$ of the electrocardiographic 
QRS complex, and end-systole was defined as the first negative crossover of the velocity curve. Offline analysis was performed using a special software program (EchoPAC 6.1.0 GE Vingmed).

Out of the cardiac cycles recorded, the RV endocardial surface was manually defined using the clearest (i.e., least myocardial artifact) of apical 4-chamber views. To train the software to track automatically the relevant myocardial motion, a region of interest was manually defined on the RV wall. Myocardial deformation was defined as longitudinal strain and strain rate was examined at basal, middle, and apical segments of the RV wall, including peak systolic displacement $(\mathrm{P})$, systolic strain peak (S), peak systolic strain rate (SRs), peak early diastolic strain rate (SRe) and late diastolic strain rate peak (SRa).

In addition, LV peak global longitudinal systolic strain was calculated from segmental averaging of the three apical long-axis views (4-chamber, 2-chamber, and apical long-axis). The LV region of interest was defined by tracking the endocardial and epicardial borders, and adjusted to accommodate wall thickness.

\section{Statistical analysis}

All analyses were performed using SPSS version 17.0 for Windows (SPSS, Chicago, IL, USA). Variables are presented as mean \pm standard deviation. One-way analysis of variance was used to assess changes among the four groups. Multiple comparisons between the groups were performed using the Student-Newman-Keuls method. Correlation coefficients, $95 \%$ confidence intervals (CIs) and percentage errors were reported. A $P$-value $<0.05$ was considered statistically significant. Intra-observer analysis of RV longitudinal strain in the 4-chamber view was conducted 2 months after completion of the
Table 2 RV echocardiographic parameters

\begin{tabular}{lcccc}
\hline & Control $(n=30)$ & $\mathrm{A}(n=30)$ & $\mathrm{B}(n=31)$ & \multicolumn{1}{c}{$\mathrm{C}(n=31)$} \\
\hline RVEF, \% & $59.75 \pm 5.62$ & $59.44 \pm 3.71$ & $55.13 \pm 3.90$ & $42.14 \pm 4.29$ \\
RVEDD, mm & $23.87 \pm 1.82$ & $24.15 \pm 1.56$ & $24.23 \pm 1.49$ & $26.20 \pm 2.13^{\mathrm{a}, \mathrm{b}, \mathrm{c}}$ \\
TAPSE, mm & $21.5 \pm 2.17$ & $19.9 \pm 2.01$ & $16.4 \pm 2.22$ & $12.2 \pm 1.98^{\mathrm{a}, \mathrm{b}, \mathrm{c}}$ \\
\hline
\end{tabular}

RVEF right ventricular ejection fraction, RVEDD right ventricular end-diastolic dimension, TAPSE tricuspid annulus peak systolic excursion. ${ }^{\mathrm{a}} P<0.01 \mathrm{cf}$. control; ${ }^{\mathrm{b}} P<0.01 \mathrm{cf}$. Group $\mathrm{A}^{\mathrm{C}}{ }^{\mathrm{C}} P<0.01 \mathrm{cf}$. Group B

initial measurements (by author MSS). For inter-observer variability, a second observer (LGS) analyzed $20 \%$ of the initial images. Intra-observer variability and inter-observer variability were assessed using the intra-class correlation coefficient (ICC) [18].

\section{Results}

\section{General characteristics and LV echocardiographic} parameters

All study subjects were in sinus rhythm at rest, and none had a history of cardiac surgery. The four groups showed an average distribution in age, body weight, height, body mass index and body surface area, which were similar among the groups $(P>0.05$; Table 1$)$. Conventional echocardiographic findings revealed that the LV dimensions, LV mass index, and greater ventricle wall thickness was significantly greater in Group $C$ compared with that of the other groups $(P<0.05)$.

\section{RV echocardiographic parameters}

The RVEDD of Group C was significantly higher than that of the other groups, and the TAPSE was significantly lower $(P<0.05$, both; Table 2$)$. Furthermore, the RVEF of Group $C$ was lower than that of the control

Table 1 Demographics and LV echocardiographic parameters

\begin{tabular}{|c|c|c|c|c|c|}
\hline & & Control $(n=30)$ & $\mathrm{A}(n=30)$ & $\mathrm{B}(n=31)$ & $C(n=31)$ \\
\hline \multirow[t]{6}{*}{ Demographics } & Age, y & $50.3 \pm 8.4$ & $51.5 \pm 6.0$ & $49.4 \pm 3.7$ & $52.3 \pm 7.6$ \\
\hline & $\mathrm{BSA}, \mathrm{m}^{2}$ & $1.56 \pm 0.26$ & $1.62 \pm 0.13$ & $1.53 \pm 0.34$ & $1.66 \pm 0.23$ \\
\hline & $\mathrm{BMI}, \mathrm{kg} / \mathrm{m}^{2}$ & $26.2 \pm 2.8$ & $25.4 \pm 3.3$ & $26.4 \pm 1.9$ & $26.1 \pm 2.1$ \\
\hline & $\mathrm{SBP}, \mathrm{mmHg}$ & $129.4 \pm 6.2$ & $120.5 \pm 11.7$ & $125.3 \pm 5.8$ & $131.5 \pm 6.7$ \\
\hline & $\mathrm{DBP}, \mathrm{mmHg}$ & $77.3 \pm 5.2$ & $79.5 \pm 5.2$ & $78.2 \pm 5.0$ & $80.3 \pm 5.2$ \\
\hline & $\mathrm{HR}$, bpm & $68.5 \pm 3.4$ & $70.5 \pm 5.6$ & $62.5 \pm 5.3$ & $67.5 \pm 2.6$ \\
\hline \multirow[t]{6}{*}{ LV parameters } & LVEF, \% & $61.20 \pm 2.86$ & $60.65 \pm 3.03$ & $58.25 \pm 5.64$ & $42.15 \pm 6.77^{\mathrm{a}, \mathrm{b}, \mathrm{c}}$ \\
\hline & $\mathrm{FS}, \%$ & $34.6 \pm 1.02$ & $33.4 \pm 2.71$ & $30.2 \pm 3.43$ & $21.6 \pm 1.25^{a, b, c}$ \\
\hline & LVDD, mm & $47.46 \pm 2.22$ & $48.94 \pm 3.40$ & $49.40 \pm 4.45$ & $60.04 \pm 3.61^{\mathrm{a}, \mathrm{b}, \mathrm{c}}$ \\
\hline & LVDs, mm & $31.01 \pm 4.47$ & $32.70 \pm 4.06$ & $33.37 \pm 4.19$ & $44.28 \pm 4.83^{\mathrm{a}, \mathrm{b}, \mathrm{c}}$ \\
\hline & IVSd, mm & $9.47 \pm 0.62$ & $9.53 \pm 0.34$ & $9.84 \pm 0.86$ & $12.01 \pm 0.45^{\mathrm{a}, \mathrm{b}, \mathrm{c}}$ \\
\hline & PWd, mm & $9.30 \pm 0.61$ & $9.37 \pm 0.50$ & $9.79 \pm 0.88$ & $11.76 \pm 0.30^{\mathrm{a}, \mathrm{b}, \mathrm{c}}$ \\
\hline
\end{tabular}

$B M I$, body mass index, BSA body surface area, DBP diastolic blood pressure, SBP systolic blood pressure, LVEF left ventricular ejection fraction, FS Left ventricular fractional shortening, $L V D D$ left ventricular end-diastolic dimension, LVDs left ventricular end-systolic dimension, IVSD Interventricular septum diastolic thickness diameter, $P W D$ posterior wall diastolic thickness diameter, ${ }^{\mathrm{a}} P<0.01 \mathrm{cf}$. control, ${ }^{\mathrm{b}} P<0.01 \mathrm{cf}$. Group $\mathrm{A}$; ${ }^{\mathrm{c}} P<0.01 \mathrm{cf}$. Group B 
Table 3 2DSTE parameters of LV peak global longitudinal systolic strain

\begin{tabular}{lclll}
\hline & Control $(n=30)$ & $\mathrm{A}(n=30)$ & $\mathrm{B}(n=31)$ & $\mathrm{C}(n=31)$ \\
\hline Basal & $-19.4 \pm 4.1$ & $-19.2 \pm 5.2$ & $-15.3 \pm 5.06^{\mathrm{ab}}$ & $-9.5 \pm 4.7^{\mathrm{abc}}$ \\
Middle & $-20.9 \pm 4.2$ & $-21.7 \pm 4.5$ & $-16.3 \pm 5.2^{\mathrm{ab}}$ & $-11.4 \pm 6.3^{\mathrm{abc}}$ \\
Apical & $-24.6 \pm 5.1$ & $-24.2 \pm 4.2$ & $-19.4 \pm 4.7^{\mathrm{ab}}$ & $-13.9 \pm 7.3^{\mathrm{abc}}$ \\
\hline
\end{tabular}

${ }^{a} P<0.05$ cf. control; ${ }^{b} P<0.05$ cf. Group $A ;{ }^{c} P<0.05$ cf. Group B

group, although this did not reach statistical significance $(P>0.05)$.

\section{DSTE parameters}

The LV longitudinal strain was less in Group B and C compared with both Group A and the control (Table 3). The average longitudinal strain and strain rate of each segment in the basal, mid, and apical regions of the RV free wall were determined in each group (Table 4). All measured echocardiographic variables in Group A were similar to that of the control group $(P>0.05)$. The SRe and $\mathrm{SRa}$ of each RV free wall segment were significantly lower in Group B than in either Group A or the control group $(P<0.05)$. However, the parameters $\mathrm{P}, \mathrm{S}$, and SRs of Groups A, B, and the control group were similar $(P>0.05)$. Notably, all the diastolic parameters and the systolic parameters $\mathrm{P}, \mathrm{S}$, and SRs were significantly lower in Group $C$ than in the other groups $(P<0.05$, all). Changes in myocardial strain within each segment (basal, mid, and apical) between the four groups are presented in Figs. 1, 2, 3 and 4.

\section{Tapse}

TAPSE was significantly lower in Group C compared with that of the other experimental groups (Figs. 5 and 6). A significant negative linear correlation was also noted between global RV systolic parameters $\mathrm{S}$ and SRs and TAPSE $\left(r_{1}=-0.84, r_{2}=-0.72\right.$, respectively, $P<0.05$; Fig. 7$)$.

Using 2D-STE to evaluate alcoholic cardiac damage, inter- and intra-observer results had good reproducibility and small variability (Table 5).

\section{Discussion}

Ventricular dysfunction is consistent with the sympathetic depressant effect of high alcohol consumption. Findings of reduced longitudinal function in the RV have been shown to reflect global ventricular dysfunction [14, 19]; further, RV dilatation and dysfunction are common findings in the pathogenesis of ACM and are closely related to the severity of LV disease [20, 21]. In addition, some studies have suggested that fat replacement of the RV in patients with ACM was likely associated with ACM [22], and that RV dysfunction was an independent predictor of cardiac death. The effect of cardiac growth on strain, changing loading conditions and ventricular contractility, myocardial fiber disarray, fat accumulation cardiac interstitial fibrosis, and edema seen in alcoholics can all affect myocardial strain.

Anatomically, the structure of the RV is more complex than that of the LV, appearing triangular when viewed from the side and crescentic when viewed in cross-section. RV myocardial fibers are three times thinner than LV fibers, making the RV myocardial wall relatively thin. The RV wall is mainly composed of two layers: the superficial layer fibers

Table 4 2DSTE parameters of RV free wall

\begin{tabular}{|c|c|c|c|c|c|}
\hline & & Control $(n=30)$ & $A(n=30)$ & $\mathrm{B}(n=31)$ & $C(n=31)$ \\
\hline \multirow[t]{3}{*}{$\mathrm{P}, \mathrm{mm}$} & Base & $21.34 \pm 1.89$ & $19.91 \pm 0.78$ & $15.14 \pm 3.32$ & $8.78 \pm 2.22^{a, b, c}$ \\
\hline & Mid & $13.61 \pm 1.14$ & $12.58 \pm 0.99$ & $10.29 \pm 2.70$ & $5.99 \pm 1.73^{a, b, c}$ \\
\hline & Apex & $5.09 \pm 0.97$ & $4.55 \pm 0.72$ & $3.36 \pm 0.63$ & $2.08 \pm 0.92^{a, b, c}$ \\
\hline \multirow[t]{3}{*}{ S, \% } & Base & $32.19 \pm 7.38$ & $26.85 \pm 2.80$ & $18.12 \pm 3.33$ & $10.71 \pm 2.71^{a, b, c}$ \\
\hline & Mid & $29.09 \pm 7.30$ & $24.46 \pm 3.09$ & $19.19 \pm 2.63$ & $12.29 \pm 4.62^{a, b, c}$ \\
\hline & Apex & $27.51 \pm 2.47$ & $25.45 \pm 2.21$ & $21.20 \pm 1.86$ & $17.53 \pm 5.02^{a, b, c}$ \\
\hline \multirow[t]{3}{*}{$\mathrm{SRS}, \mathrm{S}^{-1}$} & Base & $1.62 \pm 0.28$ & $1.29 \pm 0.10$ & $1.15 \pm 0.10$ & $0.76 \pm 0.13^{a, b, c}$ \\
\hline & Mid & $1.45 \pm 0.27$ & $1.23 \pm 0.08$ & $1.12 \pm 0.11$ & $0.87 \pm 0.13^{a, b, c}$ \\
\hline & Apex & $1.57 \pm 0.25$ & $1.28 \pm 0.18$ & $1.09 \pm 0.11$ & $0.89 \pm 0.11 \mathrm{a}, \mathrm{b}, \mathrm{c}$ \\
\hline \multirow[t]{3}{*}{ SRe, $s^{-1}$} & Base & $2.06 \pm 0.15$ & $2.00 \pm 0.14$ & $1.69 \pm 0.08^{a, b}$ & $1.35 \pm 0.11^{\mathrm{a}, \mathrm{b}, \mathrm{c}}$ \\
\hline & Mid & $1.98 \pm 0.09$ & $1.95 \pm 0.13$ & $1.71 \pm 0.07^{a, b}$ & $1.32 \pm 0.13$ \\
\hline & Apex & $2.11 \pm 0.14$ & $2.02 \pm 0.13$ & $1.82 \pm 0.07^{a, b}$ & $1.31 \pm 0.25^{a, b, c}$ \\
\hline \multirow[t]{3}{*}{ SRa, $\mathrm{s}^{-1}$} & Base & $1.58 \pm 0.16$ & $1.39 \pm 0.13$ & $1.06 \pm 0.71 \mathrm{a}, \mathrm{b}$ & $0.88 \pm 0.08 \mathrm{a}, \mathrm{b}, \mathrm{c}$ \\
\hline & Mid & $1.57 \pm 1.40$ & $1.50 \pm 0.12$ & $1.39 \pm 0.22^{a, b}$ & $0.99 \pm 0.24^{a, b, c}$ \\
\hline & Apex & $1.53 \pm 0.16$ & $1.40 \pm 0.21$ & $1.17 \pm 0.30^{a, b}$ & $0.66 \pm 0.15^{a, b, c}$ \\
\hline
\end{tabular}

$P$ peak systolic displacement, $S$ systolic strain peak, $S R s$ peak systolic strain rate, $S R e$ peak early diastolic strain rate, $S R a$ late diastolic strain rate peak. ${ }^{2} P<0.01$ cf. control, ${ }^{b} P<0.01$ cf. Group A, ${ }^{c} P<0.05$ cf. Group B 


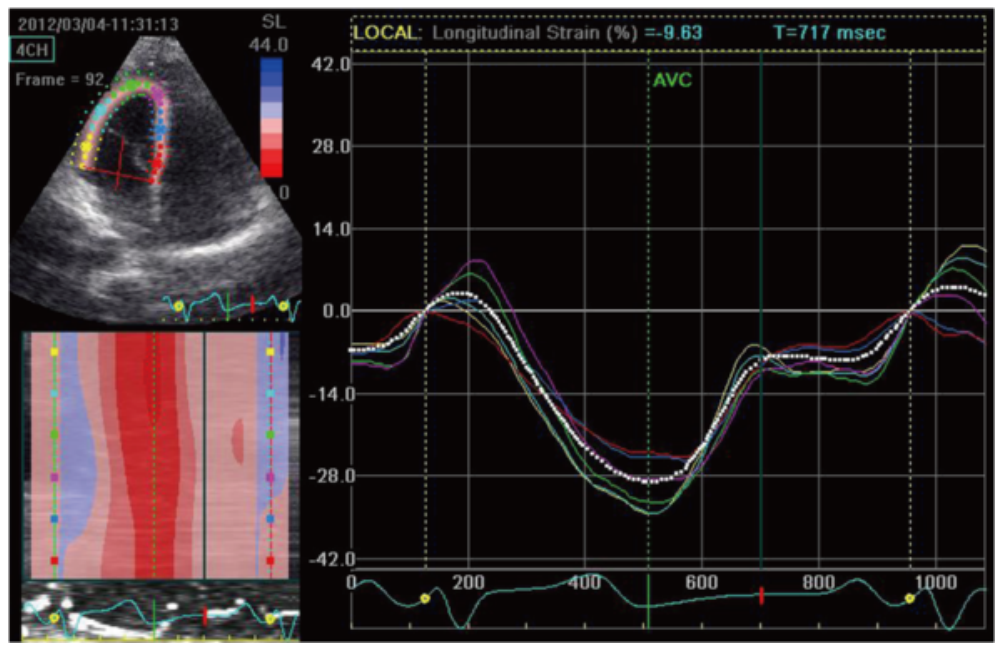

Fig. 1 Right ventricular peak systolic longitudinal strain of the control group

that are aligned circumferentially and parallel to the AV groove, and the deep layer fibers arranged longitudinally, from base to apex, which predominate RV contractile motion. Importantly, RV and LV share a common interventricular septum and the epicardial fibers are mutually encircled, thus ventricular interdependence cannot be overlooked.

Due to the complex shape of the RV, conventional transthoracic echocardiography assessment of the RV is nearly always based on qualitative estimation [23]. Thus, further studies are warranted to delineate RV function using advanced imaging techniques. Recently, a new technique has been developed for 2D-STE using B-mode images. Using this technique, myocardial deformation can be assessed by acoustic irregularities in the speckled myocardial patterns. In general, these patterns are considered stable and changes in speckle position indicate myocardial motion. Changes between the speckles are interpreted as myocardial deformation. Validation studies on 2D-STE suggest that the method is reliable and angle-independent and is not affected by translation or tethering from the surrounding tissue $[24,25]$.

To assess ventricular function in a variety of different cardiovascular diseases, 2D-STE has been confirmed to be a feasible and sensitive method [26-29]. Estimation of cardiac function by 2DSTE, without an LV geometric model, may detect abnormal RV motion prior to the occurrence of adverse systemic effects [30]. Moreover, 2D-STE is a more reliable and comprehensive assessment of myocardial function compared with tissue Doppler imaging (TDI) [31], which depends on the direction of the Doppler angle for accurate measurement.

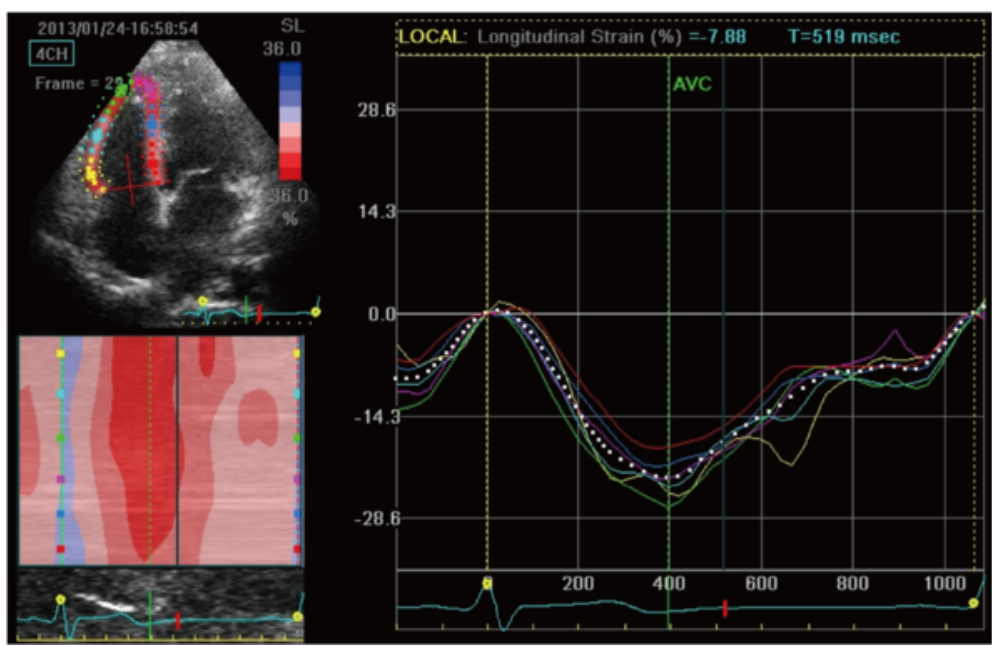

Fig. 2 Right ventricular peak systolic longitudinal strain of Group A 


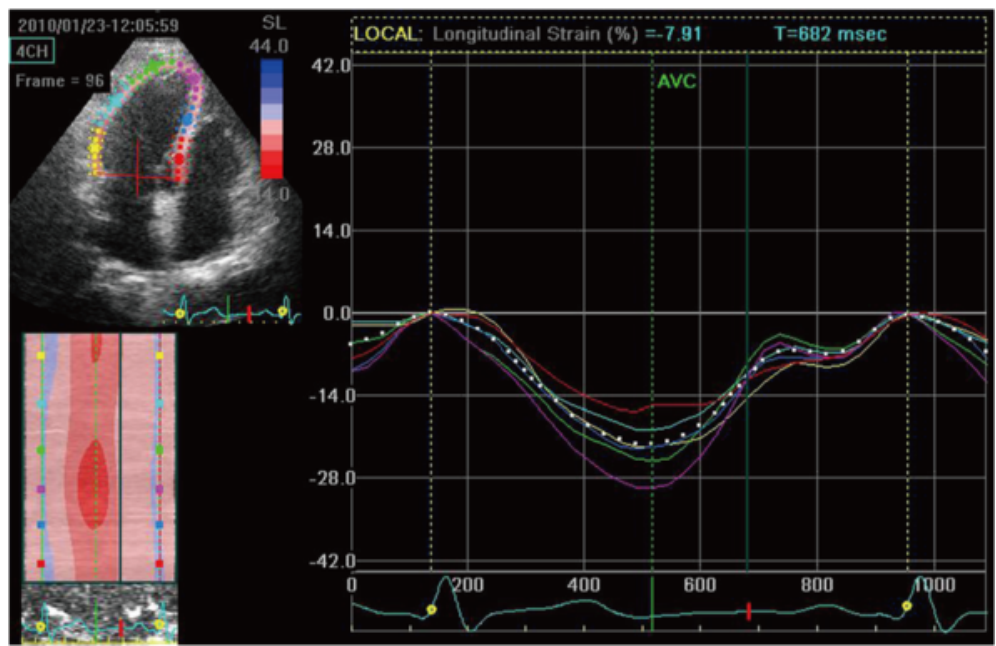

Fig. 3 Right ventricular peak systolic longitudinal strain of Group B

Because circulatory support functions of the RV are performed by longitudinal muscle, some researchers have suggested that peak longitudinal strain could be a biomarker for assessment of RV function [32]. Further, longitudinal measurements have the highest reproducibility and smallest bias, compared with the radial or circumferential dimensions [33], making longitudinal measurements a suitable approach to assess RV function. Salerno et al. [34] report the use of 2DSTE to evaluate the RV function in early dilated cardiomyopathy (DCM). Although only the RVEDD was slightly higher, 2DSTE could detect the regional RV myocardial damage. Our data is in agreement with results from this previous study on RV dysfunction.

Compared with the control group, the RVEDD of patients in Group C was significantly higher, based on conventional ultrasound parameters. However, 2D-STE analysis of Re and SRa in Group B were significantly lower than in Group A and the control, indicating altered diastolic RV function. This finding suggests that 2DSTE reflects RV function in chronic asymptomatic alcoholics more reliably as compared to conventional ultrasonic methods.

Alcoholic damage in ventricular pathology is generally considered to consist of both diastolic and systolic dysfunction. RV diastolic dysfunction is possibly related to myocardial relaxation and myocardial stiffness. Pathological changes are reflected mainly in myocardial conditions and myocardial fibrosis. Ethanol does affect tissue remodeling and causes ACM [35, 36], which is accompanied by cellular apoptosis and myocardial fibrosis [37]. In chronic asymptomatic alcoholics, levels of anti-apoptotic protein were

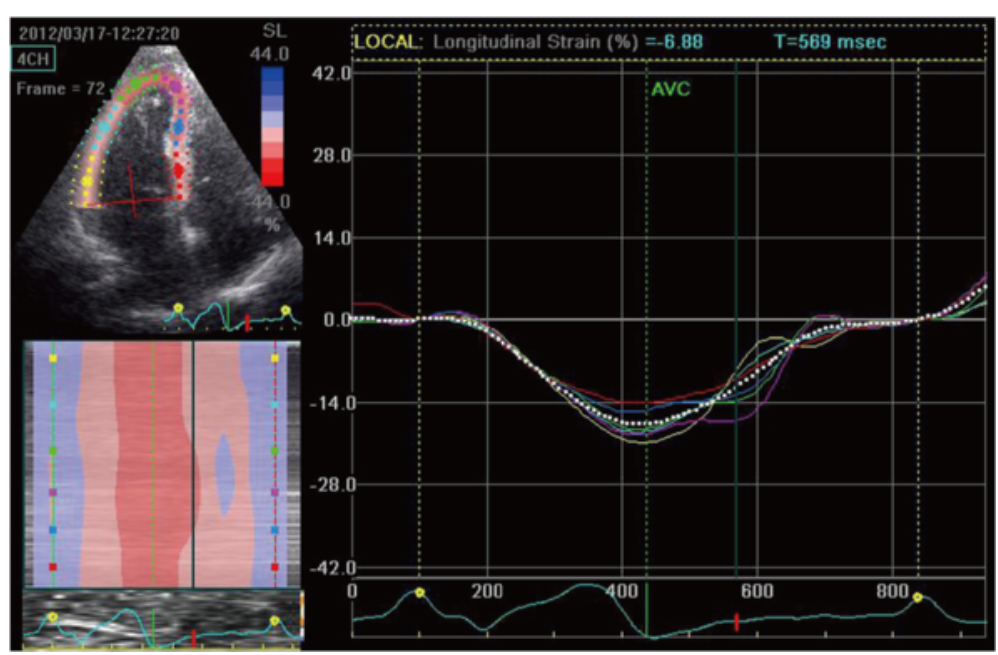

Fig. 4 Right ventricular peak systolic longitudinal strain of Group C 


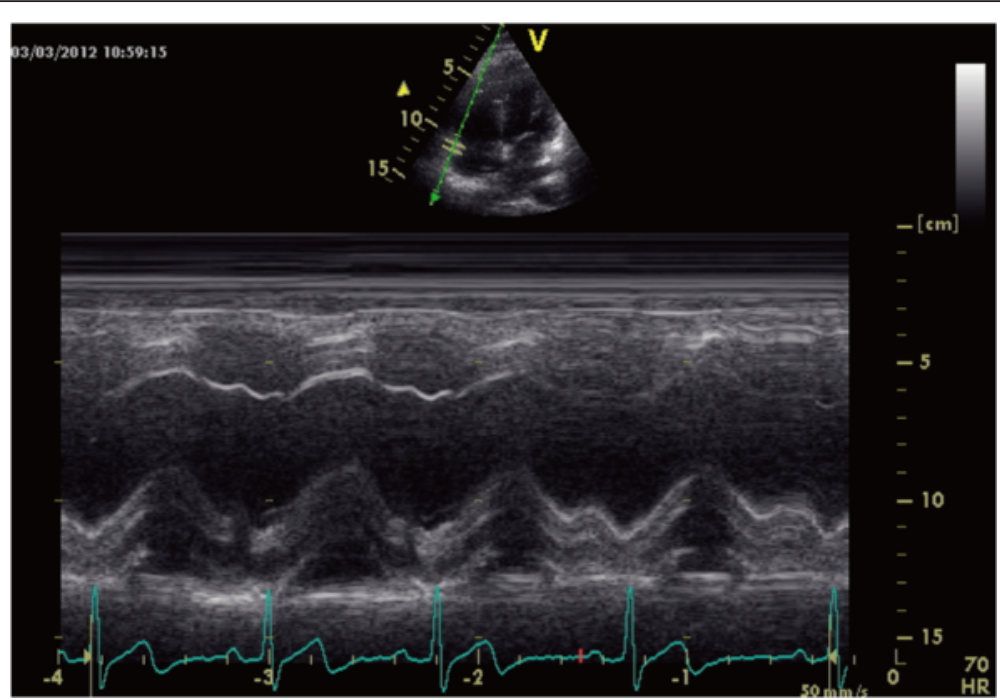

Fig. 5 TAPSE of control group

lower, and the apoptotic index higher. Therefore, the active relaxation function was impaired. Myocardial interstitial fibrosis increases myocardial stiffness, which contributes to RV dysfunction in passive relaxation, and poor prognosis of patients with DCM. Collagen production is higher in ACM patients, which may contribute to progressive ventricular dilation and dysfunction in patients with dilated cardiomyopathy [38].

Alcohol injury may cause severe cardiac dysfunction in chronic asymptomatic alcoholics [39, 40]. The present contemporary view is that ACM is manifested structurally by increased volume and hypertrophy of the LV [41], and ethanol and its metabolite acetaldehyde are thought to be cytotoxic to myocytes. The deleterious effects caused by chronic alcohol intake include activation of apoptosis, dysfunction of intracellular organelles, myofilament alteration, and disorder of intracellular calcium homeostasis, which has a key role in alcohol-induced cardiomyopathy. The deleterious effect of excess alcohol is well established in the pathogenesis of myocardial fibrosis, disruptions in the myofibrillar structure, and loss of cardiac contractility, with concomitant reduction in pump function, decompensated by lowering of cardiac output [15, 42].

Ventricular interdependence has an important role in the pathophysiology of RV dysfunction [43]. Interdependence is mostly through the common wall, the interventricular septum. Failure of the left heart could affect function of the RV, and has even been reported

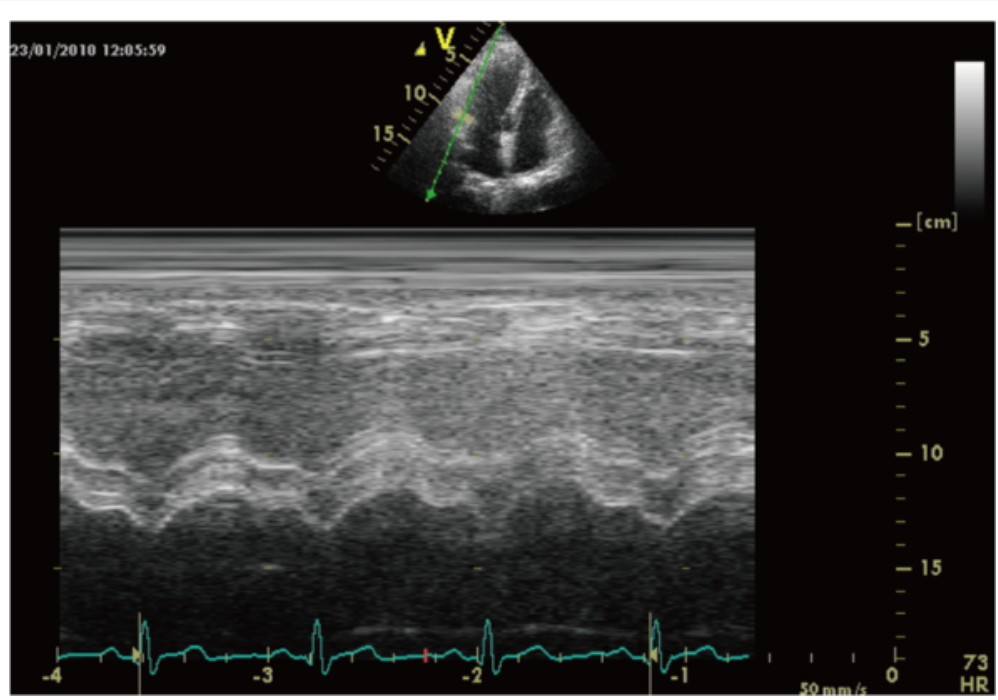

Fig. 6 TAPSE of Group C 


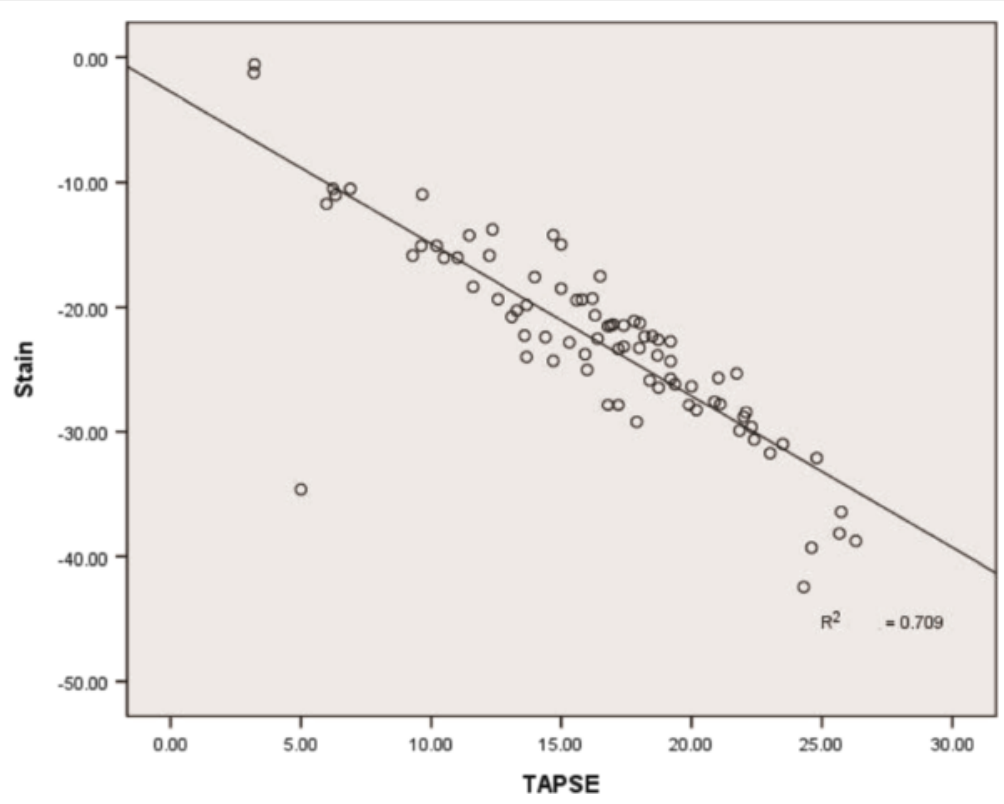

Fig. 7 Correlation between TAPSE and right ventricular peak systolic longitudinal strain

to lead to RV failure [44]. In our study, LV longitudinal strain was less in both Group B and C than Group A and the control . Less strain reflects decreased cardiac function. LV dysfunction may influence RV function and further aggravate RV failure. While few studies have reported the influence of right heart function on LV, there is consensus that RV dysfunction is a predictor of poor outcome in the setting of LV failure [45].

Because most heavy drinkers remain asymptomatic in the early stages of disease progression, early detection of morphological changes may allow early intervention in the pathogenesis of cardiovascular dysfunction [46]. The results of our study with chronic asymptomatic alcoholics suggest that changes in RV pathology may cause attenuation of myocardial function and reduction of RV longitudinal strain. As seen in our observational study, the risk of dysfunction correlates positively with the amount of alcohol consumed. Among the groups of chronic asymptomatic alcoholics in the present study, lower systolic and diastolic function of all RV free wall segments relative to the control group was most significant in Group C.

Murata et al. [47] used 2DSTE to measure longitudinal systolic myocardial strain at the base of the RV free wall and suggested that the markedly reduced longitudinal strain in the systemic RV should be interpreted as a decrease in RV myocardial contractility. In our study, consistent with other findings [48], lower longitudinal $\mathrm{P}$ in the context of reduced S and SRs of the RV free wall suggests dysfunction in this patient population. TAPSE is another reliable index of RV systolic function [49]. The significant decrease of TAPSE in Group C also can be used for evaluation of RV systolic dysfunction. In addition, we found a significant negative correlation between global RV 2DSTE parameters S, SRs, and TAPSE consistent with previous reports [50,51], which further illustrates that 2DSTE is effective as a method for detecting RV dysfunction. However, compared with 2DSTE, TAPSE is relatively load- and angle-dependent, and may be influenced by the LV. Moreover, TAPSE does not

Table 5 Inter and intra-observer analyses for RV strain

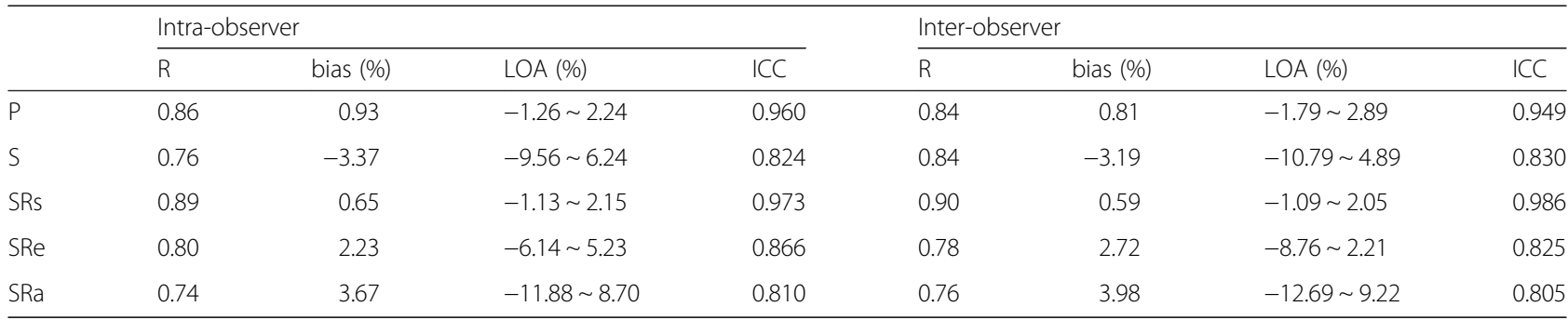

$R$, coefficient of determination, LOA limit of agreement, ICC intra-class correlation coefficient 
reflect segmental RV function and contractility [52]. 2DSTE can elucidate regional and global RV function, overcoming most of the limitations of TAPSE, because it is angle- and load-independent.

Notably, 2DSTE is strongly associated with RV contractile function confirmed by magnetic resonance imaging (MRI) [53-55]. As well, it has well-characterized specificity and sensitivity, and is a non-invasive imaging modality. Taken together, our results suggest that 2DSTE should be considered for detecting longitudinal myocardial systolic and diastolic RV function.

\section{Clinical implications}

For the assessment of RV function, 2DSTE offers improvements over conventional ultrasonic evaluation. The results of this study suggest that 2DSTE can be applied in the evaluation of RV function in chronic asymptomatic alcoholics. However, further studies are warranted to fully elucidate the clinical benefit of this imaging modality.

\section{Limitations}

The present study has several limitations. Firstly, it was conducted using a small number of male subjects, leading to uncertainty in extrapolation of these results across diverse sub-populations. Another limitation is that 2DSTE requires acceptable 2D image quality. Poor 2D image quality could confound interpretation of the results and negatively influence predictive validity of this imaging modality. In addition, several studies concluded that real-time 3-dimensional echocardiography had higher discriminating power than 2DSTE [56]. To test and verify the study, comparison with MRI and CT evaluation is necessarily. However, recent studies have shown a positive correlation between 2DSTE and MRI [53-55]. Therefore, larger studies are needed to study 2DSTE in clinical practice.

\section{Conclusions}

Alcohol may increase the risk of RV diastolic and systolic dysfunction and ethanol-induced alterations in early cardiac function have the potential to develop into ACM. Although conventional echocardiography fails to detect RV function at an early disease stage, 2DSTE is a novel and sensitive technique to assess RV function. Our study demonstrates that 2DSTE is a promising imaging modality to detect early changes in cardiac function in chronic asymptomatic alcoholics. Future confirmatory studies are warranted to further evaluate RV function using 2DSTE in this patient population.

\section{Abbreviations}

2DSTE: two-dimensional speckle-tracking echocardiography; ACM: alcoholic cardiomyopathy; DCM: dilated cardiomyopathy; EF: ejection fraction; LV: left ventricle; MRI: magnetic resonance imaging; RV: right ventricular; TAPSE: tricuspid annulus peak systolic excursion; TDI: tissue doppler imaging.

\section{Competing interests}

The authors declare that they have no competing interests.

\section{Authors' contributions}

Concept/design: LGS, MSS, GLJ; Data analysis/interpretation: MSS, LGS; Drafting article: MSS, GLJ; Critical revision of article: GLJ, MSS; Statistics: MSS, LGS; Data collection: MSS, GLJ; Approval of article: LGS, MSS, GLJ.

\section{Author details}

'Department of Ultrasound, Second Affiliated Hospital of Dalian Medical University, Dalian 116027, China. ${ }^{2}$ Department of Echocardiography, Liaoning Provincial People's Hospital, Shenyang 110000, China.

Received: 7 January 2016 Accepted: 15 April 2016

Published online: 19 April 2016

\section{References}

1. Singh $Y$, Singla V, Singh B, Rajendran R, Khandenahally RS, Manjunath CN. Quadri-chamber cardiac thrombi in alcoholic cardiomyopathy: a rare though ominous finding. Postgrad Med J. 2013;89:488-9.

2. Seiler C. Alcohol, the heart and cardiovascular system. Ther Umsch. 2000;57:200-4.

3. Ronksley PE, Brien SE, Turner BJ, Mukamal KJ, Ghali WA. Association of alcohol consumption with selected cardiovascular disease outcomes: a systematic review and meta-analysis. BMJ. 2011;342:d671.

4. Edvardsen T, Helle-Valle T, Smiseth OA. Systolic dysfunction in heart failure with normal ejection fraction: speckle-tracking echocardiography. Prog Cardiovasc Dis. 2006:49:207-14

5. Nurkalem Z, Gorgulu S, Uslu N, Orhan AL, Alper AT, Erer B, et al. Longitudinal left ventricular systolic function is impaired in patients with coronary slow flow. Int J Cardiovasc Imaging. 2009;25:25-32.

6. Mor-Avi V, Lang RM, Badano LP, Belohlavek M, Cardim NM, Derumeaux G, et al. Current and evolving echocardiographic techniques for the quantitative evaluation of cardiac mechanics: ASE/EAE consensus statement on methodology and indications: endorsed by the Japanese Society of Echocardiography. J Am Soc Echocardiogr. 2011;24:277-313.

7. Morris DA, Boldt LH, Eichstadt H, Ozcelik C, Haverkamp W. Myocardial systolic and diastolic performance derived by 2-dimensional speckle tracking echocardiography in heart failure with normal left ventricular ejection fraction. Circ Heart Fail. 2012;5:610-20.

8. Bernard Y, Morel M, Descotes-Genon V, Jehl J, Meneveau N, Schiele F. Value of speckle tracking for the assessment of right ventricular function in patients operated on for tetralogy of fallot. Comparison with magnetic resonance imaging. Echocardiography. 2014;31:474-82.

9. Sommer A, Kronborg M, Poulsen S, Bottcher M, Norgaard B, Bouchelouche $K$, et al. Empiric versus imaging guided left ventricular lead placement in cardiac resynchronization therapy (ImagingCRT): study protocol for a randomized controlled trial. Trials. 2013;14:113.

10. Dong L, Zhang F, Shu X. Clinical evaluation of left ventricle torsional function in right ventricle pressure overload individuals using twodimensional speckle tracking imaging. J Am Coll Cardiol. 2012;59:E1291.

11. Moiduddin N, Texter KM, Zaidi AN, Hershenson JA, Stefaniak CA, Hayes J, et al. Two-dimensional speckle strain and dyssynchrony in single right ventricles versus normal right ventricles. J Am Soc Echocardiogr. 2010;23:673-9.

12. Eyskens B, Ganame J, Claus P, Boshoff D, Gewillig M, Mertens L. Ultrasonic strain rate and strain imaging of the right ventricle in children before and after percutaneous closure of an atrial septal defect. J Am Soc Echocardiogr. 2006:19:994-1000

13. Kapusta L, Mainzer G, Weiner Z, Deutsch L, Khoury A, Haddad S, et al. Changes in fetal left and right ventricular strain mechanics during normal pregnancy. J Am Soc Echocardiogr. 2013;26:1193-200.

14. Pettersen E, Helle-Valle T, Edvardsen T, Lindberg H, Smith HJ, Smevik B, et al Contraction pattern of the systemic right ventricle shift from longitudinal to circumferential shortening and absent global ventricular torsion. J Am Coll Cardiol. 2007:49:2450-6.

15. Nicoll R, Henein MY. Alcohol and the heart. Alcohol Clin Exp Res. 2011;35:1737-8.

16. Levy PT, Holland MR, Sekarski TJ, Hamvas A, Singh GK. Feasibility and reproducibility of systolic right ventricular strain measurement by speckletracking echocardiography in premature infants. J Am Soc Echocardiogr. 2013;26:1201-13. 
17. Lu ZY, Zhong NS. Internal medicine. 7th ed. Beijing: People's Medical Publishing House; 2008. p. 263.

18. Shrout PE, Fleiss JL. Intraclass correlations: uses in assessing rater reliability. Psychol Bull. 1979;86:420-8.

19. Oechslin E, Jenni R. 40 years after the first atrial switch procedure in patients with transposition of the great arteries: long-term results in Toronto and Zurich. Thorac Cardiovasc Surg. 2000;48:233-7.

20. Lindqvist $P$, Henein M, Kazzam E. Right ventricular outflow-tract fractional shortening: an applicable measure of right ventricular systolic function. Eur J Echocardiogr. 2003;4:29-35.

21. La Vecchia L, Varotto L, Zanolla L, Spadaro GL, Fontanelli A. Right ventricular function predicts transplant-free survival in idiopathic dilated cardiomyopathy. J Cardiovasc Med (Hagerstown). 2006;7:706-10.

22. Hourseau M, Fornes P, Lagorce C, Lefrancq T, Martin A. Arrhythmogenic right ventricular cardiomyopathy versus fatty replacement of the right ventricle. An autopsy case report. Ann Pathol. 2002;22:469-71.

23. Rudski LG, Lai WW, Afilalo J, Hua L, Handschumacher MD, Chandrasekaran K, et al. Guidelines for the echocardiographic assessment of the right heart in adults: a report from the American Society of Echocardiography endorsed by the European Association of Echocardiography, a registered branch of the European Society of Cardiology, and the Canadian Society of Echocardiography. J Am Soc Echocardiogr. 2010;23:685-713. quiz 786-8.

24. Marwick TH. Measurement of strain and strain rate by echocardiography: ready for prime time? J Am Coll Cardiol. 2006;47:1313-27.

25. Steinhard J, Heinig J, Schmitz R, Klockenbusch W, Kiesel L. OP18. 12: Assessment of myocardial 2D strain in the right ventricle (RV) of the fetal heart by speckle tracking imaging (STI). Ultrasound Obst Gyn. 2007:30:519.

26. Migrino RQ, Aggarwal D, Konorev E, Brahmbhatt T, Bright M, Kalyanaraman B. Early detection of doxorubicin cardiomyopathy using two-dimensional strain echocardiography. Ultrasound Med Biol. 2008;34:208-14.

27. Shahul S, Rhee J, Hacker MR, Gulati G, Mitchell JD, Hess P, et al. Subclinical left ventricular dysfunction in preeclamptic women with preserved left ventricular ejection fraction: a 2D speckle-tracking imaging study. Circ Cardiovasc Imaging. 2012;5:734-9.

28. Kurt M, Tanboga IH, Isik T, Kaya A, Ekinci M, Bilen E, et al. Comparison of transthoracic and transesophageal 2-dimensional speckle tracking echocardiography. J Cardiothorac Vasc Anesth. 2012;26:26-31.

29. Ishizu T, Seo Y, Enomoto Y, Sugimori H, Yamamoto M, Machino T, et al. Experimental validation of left ventricular transmural strain gradient with echocardiographic two-dimensional speckle tracking imaging. Eur Heart J-Card Imaging. 2010;11(4):377-85.

30. Bellavia D, Pellikka PA, Dispenzieri A, Scott CG, Al-Zahrani GB, Grogan M, et al. Comparison of right ventricular longitudinal strain imaging, tricuspid annular plane systolic excursion, and cardiac biomarkers for early diagnosis of cardiac involvement and risk stratification in primary systematic (AL) amyloidosis: a 5-year cohort study. Eur Heart J Cardiovasc Imaging. 2012;13:680-9.

31. Puentes M, Mesa D, Ruiz Ortiz M, Delgado M, Mazuelos F, Suárez de Lezo J. Changes in right ventricular systolic function after transcatheter aortic valve implantation assessed by two-dimensional speckle tracking echocardiography. Rev Esp Cardiol (Engl Ed). 2012;65:964-5.

32. Kansal MM, Panse PM, Abe H, et al. Relationship of contrast-enhanced magnetic resonance imaging-derived intramural scar distribution and speckle tracking echocardiography-derived left ventricular two-dimensional strains.[J]. Eur Heart J Cardiovasc Imaging. 2012;13:152-8.

33. Koopman LP, Slorach C, Hui W, Manlhiot C, McCrindle BW, Friedberg MK, et al. Comparison between different speckle tracking and color tissue Doppler techniques to measure global and regional myocardial deformation in children. J Am Soc Echocardiogr. 2010;23:919-28.

34. Salerno G, D'Andrea A, Bossone E, Scarafile R, Riegler L, Di Salvo G, et al. Association between right ventricular two-dimensional strain and exercise capacity in patients with either idiopathic or ischemic dilated cardiomyopathy. J Cardiovascular Med. 2011;12:625-34.

35. George A, Figueredo VM. Alcoholic cardiomyopathy: a review. J Card Fail. 2011;17:844-9

36. Song YH, Li BS, Chen XM, Cai H. Ethanol extract from Epimedium brevicornum attenuates left ventricular dysfunction and cardiac remodeling through downregulating matrix metalloproteinase- 2 and-9 activity and myocardial apoptosis in rats with congestive heart failure. Int J Mol Med. 2008;21:117-24.

37. Jing L, Jin CM, Li SS, Zhang FM, Yuan L, Li WM, et al. Chronic alcohol intakeinduced oxidative stress and apoptosis: role of CYP2E1 and calpain-1 in alcoholic cardiomyopathy. Mol Cell Biochem. 2012;359:283-92.
38. Soufen $H$, Salemi V, Aneas I, Ramires F, Benício A, Benvenuti L, et al. Collagen content, but not the ratios of collagen type III/I mRNAs, differs among hypertensive, alcoholic, and idiopathic dilated cardiomyopathy. Braz J Med Biol Res. 2008:41:1098-104.

39. Movva R, Figueredo VM. Alcohol and the heart: to abstain or not to abstain? Int J Cardiol. 2013;164:267-76.

40. Matsumoto C, Miedema MD, Ofman P, Gaziano JM, Sesso HD. An expanding knowledge of the mechanisms and effects of alcohol consumption on cardiovascular disease. J Cardiopulm Rehabil Prev. 2014;34:159-71.

41. Kycina P, Murin J. Alcoholic cardiomyopathy and cardiovascular events - an insight from the Liptov region. Bratisl Lek Listy. 2013;114:337-41.

42. Rehm J, Roerecke M. Alcohol, the heart and the cardiovascular system: what do we know and where should we go? Drug Alcohol Rev. 2011:30:335-7.

43. Apostolakis S, Konstantinides $S$. The right ventricle in health and disease: insights into physiology, pathophysiology and diagnostic management Cardiology. 2012;121:263-73.

44. Walker LA, Buttrick PM. The right ventricle: biologic insights and response to disease: updated. Curr Cardiol Rev. 2013;9:73-81.

45. Polak JF, Holman BL, Wynne J, Colucci WS. Right ventricular ejection fraction: an indicator of increased mortality in patients with congestive heart failure associated with coronary artery disease. J Am Coll Cardiol. 1983:2:217-24.

46. Mackie A, Vaughan E, Verma S, Krishnamurthy P, Ramirez V, Ito A, et al. Chronic ethanol consumption impacts post-AMl cardiac function and modulates gene expression in cardiac cell types through alteration of histone 3 lysine 79 methylation. Alcohol. 2013;47:572.

47. Murata K, Ueyama T, Tanaka T, Nose Y, Wada Y, Matsuzaki M. Right ventricular dysfunction in patients with Brugada-like electrocardiography: a two dimensional strain imaging study. Cardiovasc Ultrasound. 2011;9:30.

48. Adam A, Nicholson C, Owens L. Alcoholic dilated cardiomyopathy. Nurs Stand. 2008;22:42-7.

49. Kumar V, Jose VJ, Pati PK, Jose J. Assessment of right ventricular strain and strain rate in patients with severe mitral stenosis before and after balloon mitral valvuloplasty. Indian Heart J. 2014;66:176-82

50. Ahmad H, Mor-Avi V, Lang RM, Nesser H-J, Weinert L, Tsang W, et al. Assessment of right ventricular function using echocardiographic speckle tracking of the tricuspid annular motion: comparison with cardiac magnetic resonance. Echocardiography. 2012;29:19-24.

51. Lopez-Candales A. Applicability of automated functional imaging for assessing right ventricular function. Echocardiography. 2013;30:919-28.

52. López-Candales A, Rajagopalan N, Saxena N, Gulyasy B, Edelman K, Bazaz R. Right ventricular systolic function is not the sole determinant of tricuspid annular motion. Am J Cardiol. 2006;98:973-7.

53. Singh GK, Cupps B, Pasque M, Woodard PK, Holland MR, Ludomirsky A. Accuracy and reproducibility of strain by speckle tracking in pediatric subjects with normal heart and single ventricular physiology: a twodimensional speckle-tracking echocardiography and magnetic resonance imaging correlative study. J Am Soc Echocardiogr. 2010;23:1143-52.

54. Amundsen BH, Helle-Valle T, Edvardsen T, Torp H, Crosby J, Lyseggen E, et al. Noninvasive myocardial strain measurement by speckle tracking echocardiography: validation against sonomicrometry and tagged magnetic resonance imaging. J Am Coll Cardiol. 2006;47:789-93.

55. Arnould MA, Gougnot $S$, Lemoine S, Lemoine J, Aliot E, Juilliere $Y$, et al. Quantification of right ventricular function by 2D speckle imaging and three dimensional echography. Comparison with MRI. Ann Cardiol Angeiol (Paris). 2009;58:74-85.

56. Di Bello V, Conte L, Delle Donne MG, Giannini C, Barletta V, Fabiani I, et al. Advantages of real time three-dimensional echocardiography in the assessment of right ventricular volumes and function in patients with pulmonary hypertension compared with conventional two-dimensional echocardiography. Echocardiography. 2013;30:820-8. 